https://doi.org/10.15407/ujpe65.6.552

\title{
A PROMINENT REPRESENTATIVE OF THE NEW GENERATION OF UKRAINIAN PHYSICISTS (to the 60th birthday of Ihor Mryglod)
}

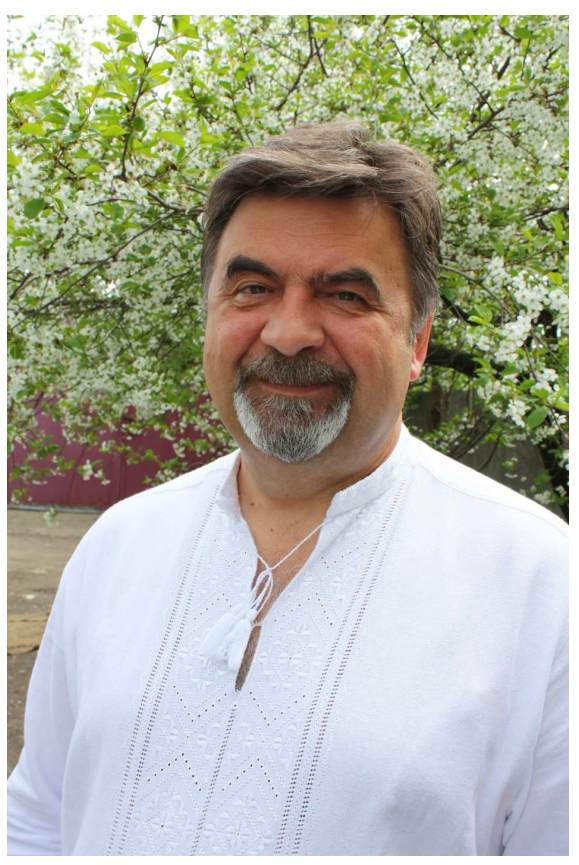

On May 26, 2020, Ihor Myronovych Mryglod a renowned scientist in the field of the statistical physics of condensed matter, Doctor of Sciences, Academician of the National Academy of Sciences of Ukraine - celebrated his sixtieth birthday. He was born in the Ternopil region of Ukraine in the family of employees. His mother's family was forcedly resettled in 1945 from the village Dobra Shlyakhetska of the Syanok district in the Lviv province just after these lands were transferred to Poland. His father's family of is indigenous to the Opillia region.

In 1982, I.M. Mryglod graduated with honour from the Physical Faculty of Ivan Franko Lviv State University. Since that time, his activity is inextricably linked with the Institute for Condensed Matter Physics (ICMP) of the National Academy of Sciences of Ukraine and with its forerunner - the Lviv Department of Statistical Physics of the Institute for Theoretical Physics of the Academy of Sciences of the Ukrainian SSR.
Ihor Mryglod is one of the prominent representatives of the Lviv school of statistical physics founded by the efforts of Academician I.R. Yukhnovskii in the 1970s. The first problem in theoretical physics put forward to Ihor Mryglod by Ihor Yukhnovskii concerned the theory of phase transitions which was very actively developed in Lviv at that time. I. Mryglod, using the method known nowadays as the Yukhnovskii's approximate renormalization-group transformation, calculated the non-universal characteristics that describe a phase transition in the $n$-component model of a structural phase transition. This made it possible to examine the common features and differences in the critical behavior at order-disorder and displacive structural phase transitions.

At the beginning of the 1990s, I.M. Mryglod acquired the method of non-equilibrium statistical operator and performed a series of studies based on this approach. In particular, having used this method for the study of generalized transport coefficients in statistical hydrodynamics of simple fluids, I.M. Mryglod together with M.V. Tokarchuk, R. Folk, and I.P. Omelyan examined non-equilibrium processes in magnetic liquids. There exists a special challenge in the study of magnetic liquids for theoreticians since it is necessary to take into account both the peculiarities of liquid state which is of classical origin and the magnetism which is of quantum origin. Moreover, the emphasis was on the non-equilibrium properties of such a system and, in particular, on the mechanisms of spin-wave formation in magnetic liquids. Theoretical studies at that time were ahead of the experiment, and a proof that they were not vain has been found only recently, when a spontaneous magnetization of magnetic nanoparticles in a solution was detected experimentally (X. Liu, N. Kent, A. Ceballos et al., Science 365 (2019) 264). In 2003, I.M. Mryglod together with M.V. Tokarchuk and Yu.K. Rudavskii were awarded the S.I. Pekar Prize of the National Academy of Sciences of Ukraine for the series of studies "Theory of dynamical properties and phase transitions in liquid magnets".

ISSN 2071-0194. Ukr. J. Phys. 2020. Vol. 65, No. 6 
Later on, Ihor Mryglod's studies led to the emergence of a new direction in non-equilibrium condensed matter physics, namely, the method of generalized collective modes. The idea of this method is to find the dynamical eigenmodes of a system by solving the generalized Langevin equation in a matrix form and by representing the time-dependent correlation functions as a separable sum of contributions, each of which is related to the corresponding collective eigenmode. Within this approach on the microscopical level, one arrives at a general picture for the dynamical behavior of fluctuations of conserved and non-conserved quantities in a fluid. This allows one to scrupulously examine a transition from the collective hydrodynamic behavior to the individual kinetics of particles. Within the framework of this approach, a number of results have been obtained for the first time, namely, the time-dependent correlation functions, the spectrum of collective excitations, and the generalized transport coefficients for a wide range of spatial and time scales for various systems (from dense noble gases and liquid metals to magnetic, polar, and ionic liquids and their mixtures). I.M. Mryglod together with T.M. Bryk have studied, in detail, the effect of fast kinetic processes on the dynamical properties of liquids, in particular, the role of excitations of the shear-wave type, the non-hydrodynamic mechanism of heat propagation, the nature of phonon-like optic modes in mixtures, the mechanisms of "fast sound" formation in binary mixtures with a large mass ratio of components.

An important peculiarity of the studies by I.M. Mryglod is a combination of the first-principle analytical calculations with the methods of computer simulations. Together with I.P. Omelyan and R. Folk, he suggested a family of new precise numerical algorithms (more than 40) for computer simulations of dynamical properties of classical and quantum systems within the framework of the molecular dynamics approach. Using an original approach to optimize the required computer time, the most effective algorithms have been found. It has been shown that, using the same computer resources, the suggested algorithms exceed the standard ones (like the Verlet, Forest, Suzuki, etc., algorithms) by a factor which varies from 10 to 100000 depending on the order of the algorithm.

Another direction of I.M. Mryglod's studies concerns the critical behavior of ionic fluids, in which the long-range Coulomb interactions play an essential role. The studies in this field, which were carried out together with O.V. Patsahan and J.-M. Caillol, led to a couple of principally new results. Thus, within the frames of the restricted primitive model of ionic fluid, it was shown for the first time that the critical behavior of ionic fluids belongs to the universality class of three-dimensional Ising model. Based on the obtained results for the Ginzburg temperature for the charge-asymmetric ionic fluid model, it turned out possible to explain, for the first time, the experimental observation of the essential effect of the relation between solvophobic and Coulomb interactions on the crossover-regime region.

Some other scientific problems are also in the field of interests of I.M. Mryglod. In particular, he (together with I.S. Bzovska) examined the basic processes of heterogeneous catalysis by using, as an example, the model reaction of the carbon monoxide oxidation on a metal surface with account for the effects of catalyst surface reconstruction. In the field of scientometrics, which is of interest to him as a researcher and as the Director of a research institution, he (in collaboration with O.I. Mryglod) carried out a couple of studies. In particular, he suggested a mathematical model of how a paper passes in a scientific periodic journal, analyzed Ukrainian periodic research journals and proposed some recommendations in this regard. Within the problems related to the Chornobyl disaster, he (in collaboration with M.V. Tokarchuk and I.P. Omelyan) performed pioneer studies on the physical and chemical properties and the state of lava-like fuel-containing materials in the object "Shelter" as well as regarding their changes in time. In particular, he described the dust appearance and showed the peculiarities of submicron dust dynamics. These studies urged important experiments that allow one to amend the program of monitoring the lava-like fuel-containing materials and to work out recommendations how to process them at various stages of transforming the object "Shelter" into an ecologically safe system.

Ihor Mryglod, who is the Director of the Institute for Condensed Matter Physics of the National Academy of Sciences of Ukraine since 2006, is a disciple of Academician I.R. Yukhnovskii, the founder and creator of the Institute (predecessor); he is also the successor of Academician I.R. Yukhnovskii in directing the Institute. During his directorship, the Insti- 
tute has grown to a modern effective research center, which is well known in Ukraine and over the world. A series of initiatives proposed by Ihor Mryglod has been in favor of this. In particular, one has to mention the construction of the ICMP computation cluster which was launched about twenty years ago and which was the first cluster in the National Academy of Sciences of Ukraine. This was a very effective and far-sighted investment which allows the Institute over many years to efficiently perform computer simulations of various condensed matter objects, to be an active participant of the Ukrainian National Grid and the Program of informatization, to become an important computer resource center in Ukraine. I.M. Mryglod plays an important role as an Associate Editor of the journal Condensed Matter Physics. He carefully follows the tendencies in the field of scientific periodic journals and scientometrics: The journal was far in advance to introduce the digital identificators DOI; created bibliometric data base, which allows one to quantitatively analyze the publication activity; took a direction toward making the journal more visible; introduced the electronic editorial processing. Over many years, the journal is among the first three research journals of Ukraine with the highest impact factor.

The Institute for Condensed Matter Physics regularly organizes large conferences under the guidance of I.M. Mryglod. Thus, in the 2000s on the initiative of I.M. Mryglod, the traditional conferences on statistical physics, which were launched by I.R. Yukhnovskii in the 1970 s, were revived. The fifth conference from this series took place last year; it was dedicated to the 110th anniversary of M.M. Bogolyubov's birth and to the 50th anniversary of the foundation of the first department of the Institute. One should mention the Annual Workshop and Award for young researchers in the field of statistical physics and condensed matter theory, which was also once initiated by Ihor Mryglod, and now it is successfully run by young researchers of the Institute with his personal support. This year, when I.M. Mryglod observes his 60 th birthday, it will be the 20th Workshop.

Academician I.M. Mryglod is an active participant of discussions and informal interested communities that are concerned with the reformation of science in Ukraine. He took part in the preparation of the bill "About scientific and scientific-technical activity", a number of the elaborated issues were taken into account within the law adopted by the Parliament of Ukraine. He realized his vision regarding a further development of science in Ukraine carrying on several scientific and organizational duties, in particular, as a member of the Council of the State Fund for Basic Researches, the Deputy Academician Secretary of the Department of Physics and Astronomy of the NAS of Ukraine, a member of the expert group at the Committee for Science and Education of the Parliament of Ukraine, the Deputy Head of the Western Scientific Center of the NAS and the MES of Ukraine, a member of the steering board of the Lviv City Council Program "Lviv Scientific," etc. As a well-known specialist in condensed matter physics, I.M. Mryglod is a member of the Editorial Boards of several research journals: Open Physics (previously "Central European Journal of Physics"), Physical Collection of the Shevchenko Scientific Society, Bulletin of the National University "L'viv'ska Politekhnika". He also acts as an active referee for top research journals such as Scientific Reports, Physical Review Letters, Physical Review E, Journal of Chemical Physics.

I.M. Mryglod enjoys great respect among the scientific community, his colleagues appreciate his concern and advice. He has been awarded by Honors of the NAS of Ukraine "For scientific achievements" and "For preparation of a new generation of scientists", Diploma of honor of the Parliament of Ukraine, the Order of Merit of the third degree.

Friends and colleagues warmly congratulate Ihor Mryglod on the jubilee, wish him new achievements in physics, realization of ideas in the organization of science in Ukraine, and raising the prestige of the work of a scientist in Ukraine.

I.R. YUKHNOVSKII, V.G. BAR'YAKHTAR, A.S. BAKAI, V.M. LOKTEV, YA.S. YATSKIV, A.G. ZAGORODNY, T.M. BRYK, O.V. DERZHKO, R. FOLK, YU.V. HOLOVATCH, M.F. HOLOVKO, O.L. IVANKIV, M.P. KOZLOVSKII, O.V. PATSAHAN, M.V. TOKARCHUK 